\title{
Evidence of Mirror Neurons in Human Inferior Frontal Gyrus
}

\author{
James M. Kilner, ${ }^{1}$ Alice Neal, ${ }^{1}$ Nikolaus Weiskopf, ${ }^{1}$ Karl J. Friston, ${ }^{1}$ and Chris D. Frith ${ }^{1,2}$ \\ ${ }^{1}$ The Wellcome Trust Centre for Neuroimaging, Institute of Neurology, UCL, London WC1N 3BG, United Kingdom, and Niels Bohr Project "Interacting \\ Minds," Center of Functionally Integrative Neuroscience, University of Aarhus, 8000 Århus, Denmark
}

There is much current debate about the existence of mirror neurons in humans. To identify mirror neurons in the inferior frontal gyrus (IFG) of humans, we used a repetition suppression paradigm while measuring neural activity with functional magnetic resonance imaging. Subjects either executed or observed a series of actions. Here we show that in the IFG, responses were suppressed both when an executed action was followed by the same rather than a different observed action and when an observed action was followed by the same rather than a different executed action. This pattern of responses is consistent with that predicted by mirror neurons and is evidence of mirror neurons in the human IFG.

\section{Introduction}

Mirror neurons were first discovered in the premotor area F5 of macaque monkeys (di Pellegrino et al., 1992; Gallese et al., 1996; Rizzolatti et al., 2001; Umiltà et al., 2001) and subsequently in inferior parietal lobule, area PF (Gallese et al., 2002; Fogassi et al., 2005). Mirror neurons discharge not only during action execution but also during action observation. Since the discovery of mirror neurons, a number of neuroimaging studies have claimed that a mirror neuron system exists in humans and that homologous areas in the human brain are activated when observing and executing movements (Rizzolatti et al., 1996; Decety et al., 1997; Buccino et al., 2001; Grèzes and Decety, 2001; Gazzola and Keysers, 2009). However, over a decade after their discovery, there is still debate as to whether any of the human neuroimaging studies constitute conclusive evidence for mirror neurons in the human homolog of area F5, the inferior frontal gyrus (IFG) (Dinstein et al., 2008). To date, the best evidence for mirror neurons in the human IFG is the demonstration that there is a significant spatial overlap between activity in this region during both action observation and execution (Rizzolatti et al., 1996; Decety et al., 1997; Buccino et al., 2001; Grèzes and Decety, 2001). The problem with this evidence is that the majority of neurons active during either action execution and observation are not mirror neurons (Rizzolatti and Craighero, 2004). Therefore, the fact that a volume of cortex in IFG has an increased blood oxygenation level-dependent (BOLD) signal during observation and execution of an action does not necessarily mean that the same neurons are active in both conditions (Dinstein et al., 2007, 2008). One approach to attribute the functional magnetic resonance imaging (fMRI) response to a single neuronal population is adaptation, or repeti-

Received May 27, 2009; revised July 7, 2009; accepted July 8, 2009.

J.M.K., A.N., N.W., K.J.F., and C.D.F. were funded by the Wellcome Trust, UK.

Correspondence should be addressed to James M. Kilner, The Wellcome Trust Centre for Neuroimaging, Institute

of Neurology, UCL, 12 Queen Square, London WC1N 3BG, UK. E-mail: j.kilner@fil.ion.ucl.ac.uk.

DOI:10.1523/JNEUROSCI.2668-09.2009

Copyright $\odot 2009$ Society for Neuroscience $\quad$ 0270-6474/09/2910153-07\$15.00/0 tion suppression. The logic of this approach is that as stimuli that evoke activity in a specific neuronal population are repeated, the magnitude of the response decreases or adapts (GrillSpector et al., 2006; Dinstein et al., 2007, 2008; Dinstein, 2008). fMRI adaptation effects have been reported previously both when the observed actions are repeated (Hamilton and Grafton, 2006, 2008; Dinstein et al., 2007) and when the executed actions are repeated (Dinstein et al., 2007) [unimodal repetition suppression (umRS)]. Areas of the cortex that contain mirror neurons should show adaptation both when an action is executed and subsequently observed and when an action is observed and subsequently executed [cross-modal repetition suppression (xmRS)]. Three previous fMRI studies have failed to demonstrate significant xmRS in the human IFG (Dinstein et al., 2007; Chong et al., 2008; Lingnau et al., 2009) (see also Grafton, 2009).

Here we used an fMRI adaptation paradigm that was designed to be maximally sensitive to xmRS in the IFG. Using this paradigm, we were able to show significant xmRS effects in human IFG, at the single-subject level, at the group level using a region of interest (ROI) analysis and also at the group level using a typical whole-brain analysis. These results are consistent with the existence of mirror neurons in human IFG.

\section{Materials and Methods}

Data were recorded from 10 healthy right-handed subjects ( 7 females, 25-45 years). All subjects gave written informed consent before testing, and the recordings had local ethical committee approval.

The task and scanning paradigms used in this study were designed to increase the probability of activity in human IFG. Subjects were asked to either observe or execute one of two actions performed by the right hand-a precision grip or an index finger pull. These actions were chosen for two reasons. First, the three previous studies that failed to show cross-modal repetition suppression effects in the IFG used meaningless or pantomime actions (Dinstein et al., 2007; Chong et al., 2008; Lingnau et al., 2009). Studies of mirror neurons in area F5 of the macaque have focused on goal-directed actions. Indeed, mirror neurons in macaque monkeys do not even respond to mimed actions (Umiltà et al., 2001). In light of this, we built an fMRI-compatible manipulandum that allowed 
the subject to make two different grip actions. The actions were made in a way that required only movements around the wrist and the hand, thus minimizing movement artifacts in the fMRI data. The second reason these actions were chosen was that previous research has shown that the discharge of neurons in area F5 of the macaque monkey is modulated by the nature of the grip used during action execution (Gentilucci et al., 1988; Rizzolatti et al., 1988; Jeannerod et al., 1995). We reasoned that tasks that are known to selectively activate different populations of neurons within the IFG would increase our chances of seeing repetition suppression.

In the scanner, on each trial, subjects were presented with pairs of stimuli sequentially. These pairs could consist of two executions, two observations, or mixed execution and observation conditions. During the observation conditions, subjects observed a video of one of the actions-index finger ring pull and precision grip. In total there were 28 different exemplar videos of each movement made by two different actors, one female and one male. Each video lasted $\sim 750 \mathrm{~ms}$. Subjects were required to execute an action when a red arrow appeared on the screen that pointed toward the object that they were required to move. The arrow either pointed up the screen-indicating the index finger ring pull-or left-indicating the precision grip (Fig. 1). Subjects were trained before scanning to execute the correct action as rapidly as possible following the imperative cues. These imperative cues were presented for $500 \mathrm{~ms}$. Trials were categorized into eight types: execute-execute same, execute-execute different, observe-observe same, observe-observe different, execute-observe same, execute-observe different, observe-execute same, and observe-execute different. Within each trial, there was a $500 \mathrm{~ms}$ gap between presentations of the two stimuli, and there was a between-trial jittered "wait" with a mean of 5000 $\mathrm{ms}$ (SD $1000 \mathrm{~ms}$ ). A rapid interstimulus interval was chosen as the time constant of the neuronal repetition effects in IFG are not known. Subjects performed four sessions, where each session consisted of 96 trials with each of the eight trial types presented 12 times.

We acquired T2*-weighted echo-planar images (EPIs) with BOLD contrast on a $3 \mathrm{~T}$ whole-body MRI scanner (Magnetom TIM TRIO, Siemens Medical) operated with the standard 12-channel head receive and body transmit coil. A total of 600 volumes were collected for each of the four sessions; these included six dummy volumes at the start of each session to allow for T1 equilibration. Each volume consisted of 16 slices that were positioned before scanning to cover left and right IFG (repetition time $1072 \mathrm{~ms}$ ). Imaging parameters were as follows: in-plane resolution $3 \times 3 \mathrm{~mm}^{2}$, slice thickness $2 \mathrm{~mm}$ with $1 \mathrm{~mm}$ interslice gap, echo time $30 \mathrm{~ms}, 64 \times 64$ matrix. High-resolution T1-weighted structural scans (MDEFT) (Deichmann et al., 2004) were collected for each subject and were coregistered to their mean EPI images.

Data preprocessing of the EPI functional scans, including spatial realignment, normalization to a standard EPI template, and smoothing with a $4 \mathrm{~mm}$ (full-width at half-maximum) Gaussian kernel, using SPM5 (www.fil.ion.ucl.ac.uk/spm). The event-related fMRI data were then analyzed using a linear convolution model in the usual way: Stimulus functions comprised a set of delta functions corresponding to the onset times of the different conditions. The first stimulus of each within-trial pair was modeled by two regressors, one for a pair beginning with an execution condition and one for a pair beginning with the observation condition. For the second within-trial stimulus, there were eight different stimulus functions depending upon the trial category (see above). In addition, the "wait" period that occurred between every pair of stimuli was explicitly modeled. These functions were convolved with a canonical hemodynamic response function for explanatory variables or regressors. Subjectspecific movement parameters and drift terms (high-pass filter cutoff, $128 \mathrm{~s}$ ) were also modeled as covariates of no interest. Condition-specific estimates of neural activity (betas), corresponding to the amplitude of the modeled response, were computed at each voxel for each subject. The contrasts of interest in this study are execution $>$ wait (where execution is the regressor depicting the onset of the execution conditions when execution was the first of the pair), observation $>$ wait (where observation is the regressor depicting the onset of the observation conditions when observation was the first of the pair), and xmRS same $<$ different (where the repeated action is less than the different action in mixed or cross-modal trials). All voxels reported conform to MNI (Montreal Neurological Institute) coordinate space. For display, the right side of the image corresponds to the right side of the brain. All analyses were restricted to left and right IFG using a small volume search. This volume was created from the maximal extent of peak activations reported in the meta-analysis in Table 1 of Dinstein et al. (2007). This is depicted graphically in Figure $2 D$ (red boxes). For display purposes, activations in the whole of the IFG are shown this area is shown graphically in Figure $2 D$ (yellow box). In this study it was not appropriate to use a region of interest based on published cytoarchitectonic maps. Some previous studies have used an ROI analysis approach based in the probabilistic maps of BA44. However, the human homolog of area F5 is known not to be restricted to BA44 but also includes ventral BA6 (Morin and Grèzes).

\section{Results}

An initial analysis focused on the simple effects of increases in BOLD during action execution and observation. In agreement with previous literature, all subjects showed significantly greater activity in both left and right IFG when subjects either executed or observed an action compared with rest ( $p<0.05$ familywise error corrected) (see Fig. 2 for an example from a single subject). Furthermore, the conjunction of these contrasts showed that there was significant overlap between voxels active for action execution and observation in each subject in both left and right IFG (Fig. 2 for single subject and Table 1 for all subjects).

\section{Group-level analysis: ROI approach}

Due to the failure of three previous studies that used group-level analysis to show significant xmRS effects in the IFG (Dinstein et al., 2007; Chong et al., 2008; Lingnau et al., 2009), in a first analysis of xmRS, the critical group-level comparisons were performed on data extracted from the single-subject data. To determine whether there was significant xmRS, we performed a $2 \times 2$ repeated-measures ANOVA at the between-subject level on the estimated responses to the second stimulus of each trial pair. These responses were during execute-observe same, executeobserve different, observe-execute same, and observe-execute different trials, in left and right IFG. These regions were identified 
A
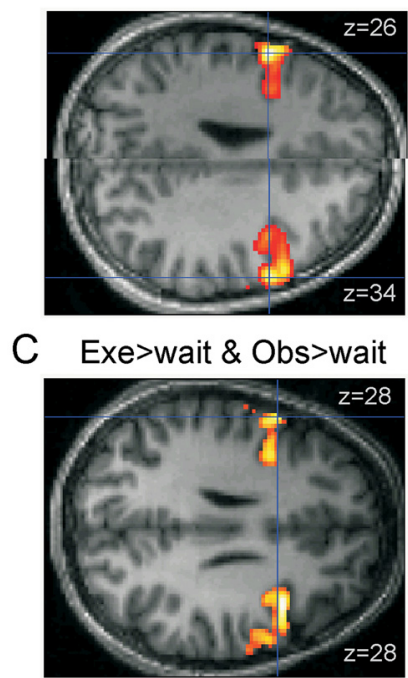

E

xmRS same<diff

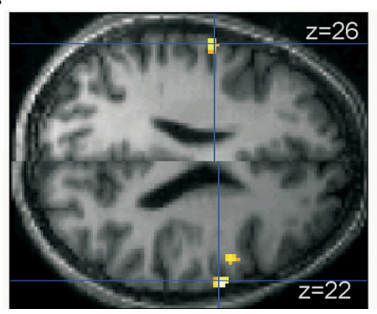

B
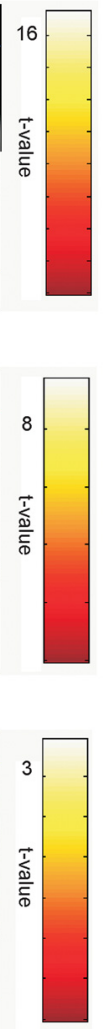

D

$\mathrm{F}$
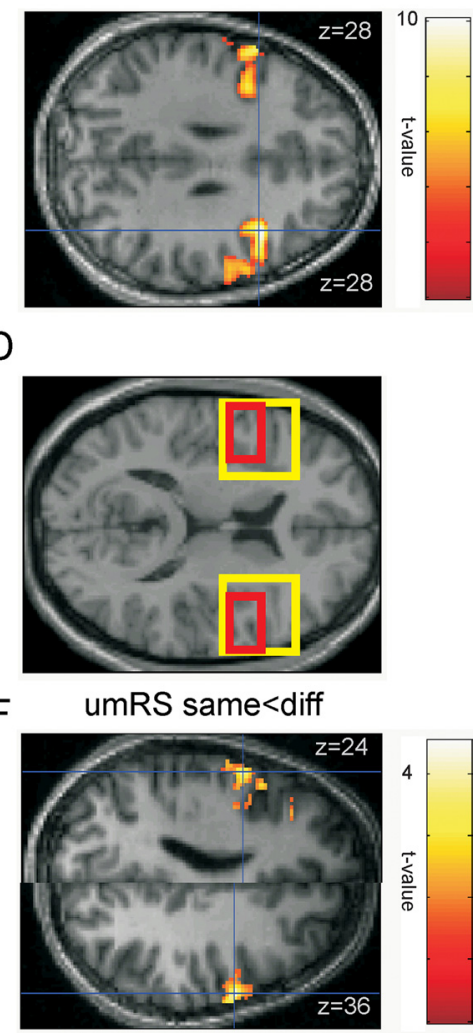

Figure 2. Single-subject data. $\boldsymbol{A}-\boldsymbol{C}, \boldsymbol{E}, \boldsymbol{F}$, SPMs of the $t$ values from a single subject, subject 7, for the different contrasts of interest. This subject was the median subject based upon the peak $t$ value for the xmRS same $<$ diff contrast. All the SPM search volumes (shown in $D$ by a yellow box) were restricted to the IFG but are shown on the whole brain. All further analysis was restricted to posterior IFG corresponding to a complex of BA44 and ventral BA6. This is shown in $D$ by a red box. $A, B$, SPM showing where the BOLD activity is greater during action execution than during the wait period $(\boldsymbol{A})$ and where the BOLD activity is greater during action observation than during the wait period $(\boldsymbol{B})$. $\boldsymbol{C}$, Voxels that were conjointly active during both action observation and action execution. The SPMs in $\boldsymbol{A}-\boldsymbol{C}$ are shown thresholded at $p<0.001$ (uncorrected). $\boldsymbol{E}, \boldsymbol{F}$, Regions of IFG with an xmRS effect $(\boldsymbol{E})$ and with a umRS effect $(\boldsymbol{F})$. These SPMs are thresholded at $p<0.05$ (uncorrected). In $\boldsymbol{A}-\boldsymbol{C}, \boldsymbol{E}$, and $\boldsymbol{F}$, the SPM is shown for the axial slice at maximal activity in the left and right hemispheres separately.

with an orthogonal contrast; the conjunction between action execution and action observation. We used the estimates from the peak voxel of each subjects SPM, which is equivalent to taking a local weighted average as the data were smoothed with a Gaussian kernel. It should be noted that this is an unbiased method as the ROI was defined from a fully balanced orthogonal contrast (as prescribed in Kriegeskorte et al., 2009). The within-subject factors were therefore congruence (same or different) and order (executed then observed or observed then executed). The analysis of responses in left IFG showed a significant effect of congruence $\left(F_{(1,9)}=20.888, p<0.001\right)$ and $\operatorname{order}\left(F_{(1,9)}=93.657, p<\right.$ $0.001)$, but no significant interaction between the two $\left(F_{(1,9)}=\right.$ $0.068, p=0.801$ ) (Fig. 3). Importantly, a post hoc $t$ test revealed that this effect was driven by repetition suppression effects, both when the action was first executed and then observed and when the action was observed and then executed $(p<0.05)$. The equivalent analysis in right IFG showed only a significant main effect of order $\left(F_{(1,9)}=29.71, p<0.001\right)$. The main effect of congruence $\left(F_{(1,9)}=0.481, p=0.505\right)$ and the interaction $\left(F_{(1,9)}=0.720, p=0.418\right)$ were not significant. The absence of a significant congruence effect in the right hemisphere does not mean that there are no repetition suppression effects in the right hemisphere, just that these effects were not consistent across subjects at the peak conjunction of action execution and action observation.

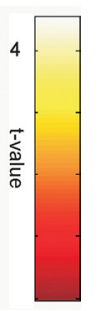

\section{Group-level and single-subject} analyses: non-ROI approach

The previous analysis assumes that $\mathrm{xmRS}$ occurs at the peak of the conjunction of observing an action and executing an action. This is sensible, given that the minimum requirement for mirror neurons is that the area activated should be activated by both action observation and execution. However, the converse is not true. In other words, maximal xmRS does not necessarily have to be at the peak of responses to observation and execution. To address this, we characterized the xmRS at both the group level and in each individual subject (Figs. 3, 4, Table 2; supplemental Fig. 1, available at www.jneurosci.org as supplemental material). At the group level, only two clusters survived a liberal statistical threshold $(p<0.05$ uncorrected). This liberal threshold was chosen to demonstrate the spatial specificity of the effect (Fig. 4). However, although a liberal threshold was chosen to show spatial specificity, the peak voxel within the left IFG was significant at the required conservative threshold $(p<0.05$ corrected for small volume correction for left IFG; $t=6.35$ at $-50,-2,12)$. In other words, at the group level, there was a significant xmRS in the left IFG that had high spatial specificity to the left IFG. In addition, at the single-subject level, 7 of 10 subjects showed an xmRS effect $(p<$ 0.001 uncorrected) in either left or right IFG. Importantly, the peak voxel in left IFG for the xmRS contrast was also significant during action execution and action observation $\left(t_{(9)}=3.23, p<0.05 ; t_{(9)}=2.98, p<0.05\right)$. This demonstrates that the area of cortex that was significantly modulated by xmRS also shows the basic properties of mirror neurons; namely, it is significantly activated during both action execution and observation. In summary, here we have shown significant xmRS effects in the IFG at the group level using a region-of-interest analysis, at the group level using a whole-brain SPM, and at the single-subject level.

\section{Spatial differences in peak responses}

In addition, we noticed that the locations of the peaks for the three contrasts of interest, execution, observation, and xmRS, were not at the same coordinates within IFG (Tables 1, 2, Fig. 5). Furthermore, the different contrasts peaked in systematically different locations across subjects. These small yet significant differences were observed in both the left and right IFG (Fig. 5). On average the peak execution effect was $6.7 \mathrm{~mm}$ more lateral than the corresponding peak for action observation (left IFG, $4.2 \mathrm{~mm}$; right IFG, $9.2 \mathrm{~mm}$ ). A $2 \times 2$ repeated-measures ANOVA on the locations, where the factors were condition (execution or observation) and hemisphere (left or right), revealed only a significant effect of condition $\left(F_{(1,9)}=10.048, p=0.011\right)$. Similarly, the location of the peak for the xmRS effect was on average 4.4 $\mathrm{mm}$ more ventral than the corresponding peaks for the observation conditions (left IFG, $5 \mathrm{~mm}$; right IFG, $3.8 \mathrm{~mm}$ ). A $2 \times 2$ 
Table 1. Conjunction: exe $>$ wait and obs $>$ wait

\begin{tabular}{|c|c|c|c|c|c|c|c|c|c|c|}
\hline \multirow[b]{3}{*}{ Subject } & \multicolumn{5}{|c|}{ Left IFG } & \multicolumn{5}{|c|}{ Right IFG } \\
\hline & \multicolumn{3}{|c|}{ Peak MNI coordinates } & \multirow[b]{2}{*}{$p$ value } & \multirow[b]{2}{*}{$t$ value } & \multicolumn{3}{|c|}{ Peak MNI coordinates } & \multirow[b]{2}{*}{$p$ value } & \multirow[b]{2}{*}{$t$ value } \\
\hline & $x$ & $y$ & $Z$ & & & $x$ & $y$ & $Z$ & & \\
\hline 1 & -38 & 2 & 36 & $<0.001$ & 9.62 & 44 & 12 & 32 & $<0.001$ & 9.1 \\
\hline 2 & -56 & 8 & 24 & $<0.001$ & 5.31 & 48 & 12 & 22 & $<0.001$ & 5.6 \\
\hline 3 & -62 & 8 & 16 & 0.012 & 2.25 & 40 & 6 & 24 & $<0.001$ & 9.96 \\
\hline 4 & -56 & 4 & 12 & $<0.001$ & 3.86 & 42 & 12 & 24 & $<0.001$ & 3.87 \\
\hline 5 & -56 & 8 & 32 & $<0.001$ & 11.66 & 46 & 8 & 30 & $<0.001$ & 11.71 \\
\hline 6 & -60 & 8 & 16 & $<0.001$ & 20.82 & 54 & 4 & 26 & $<0.001$ & 20.09 \\
\hline 7 & -58 & 10 & 28 & $<0.001$ & 8.01 & 40 & 12 & 28 & $<0.001$ & 9.71 \\
\hline 8 & -58 & 0 & 18 & $<0.001$ & 12.86 & 60 & 6 & 32 & $<0.001$ & 11.72 \\
\hline 9 & -60 & 6 & 30 & $<0.001$ & 4.06 & 42 & 10 & 30 & $<0.001$ & 11.47 \\
\hline 10 & -60 & 8 & 26 & $<0.001$ & 6.15 & 48 & 4 & 32 & $<0.001$ & 4.99 \\
\hline
\end{tabular}
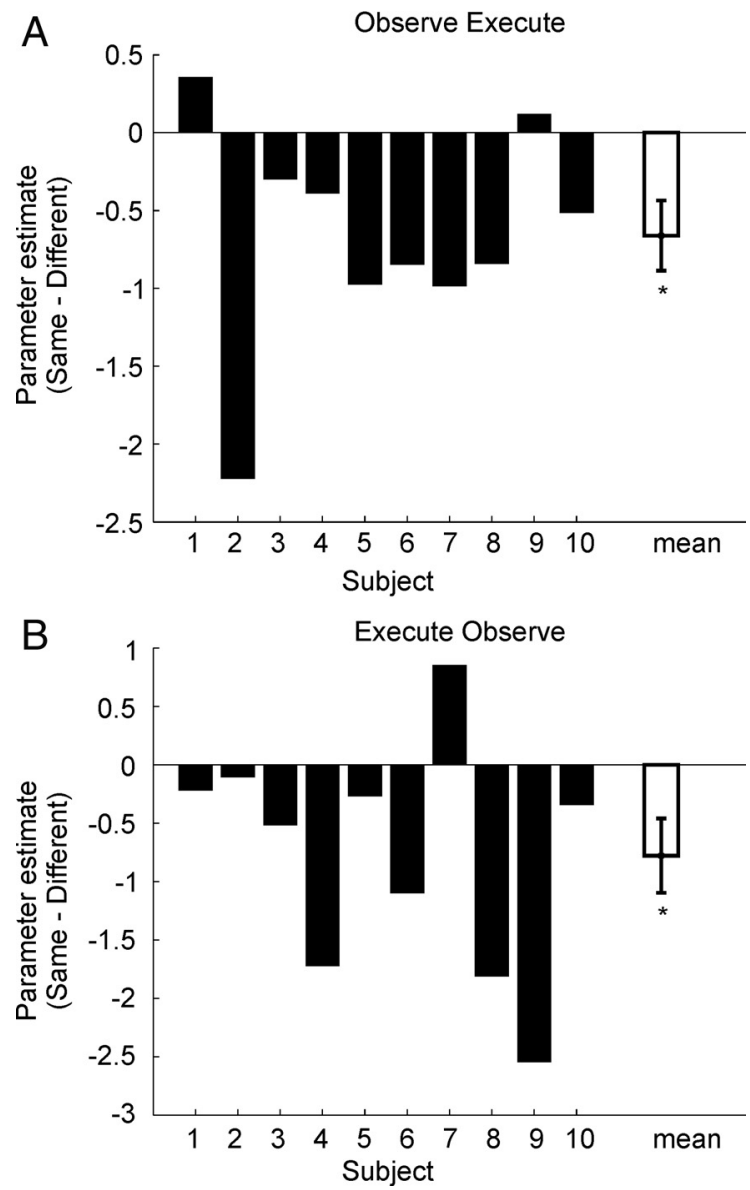

Figure 3. $x m R S$ effects. $\boldsymbol{A}$, The parameter estimates for each of the 10 subjects (black bars) for the $x m R S$ when subjects first observed and then executed an action. In 8/10 subjects, the parameter estimate was less when subjects executed the same action compared with a different action. The mean parameter estimate across all subjects is shown in white, and error bars show the SE. $\boldsymbol{B}$, The same data as in $\boldsymbol{A}$ but for pairs in which the subjects first executed and then observed an action. Here $9 / 10$ subjects show a repetition suppression effect. In both cases the parameter estimate was taken at the voxel that corresponded to the peak $t$ value for the conjunction of unimodal execution $>$ wait and observation $>$ wait in the left IFG (this voxel selection was orthogonal to any differences between cross-model trials). ${ }^{*}$ indicates significance at $p<0.05$.

repeated-measures ANOVA on the locations, where the factors were condition (xmRS or observation) and hemisphere (left or right) revealed only a significant effect of condition $\left(F_{(1,9)}=\right.$ 9.991, $p=0.012$ ). These results suggest that the locations of the peak effects are in different areas of IFG; with the peak elicited
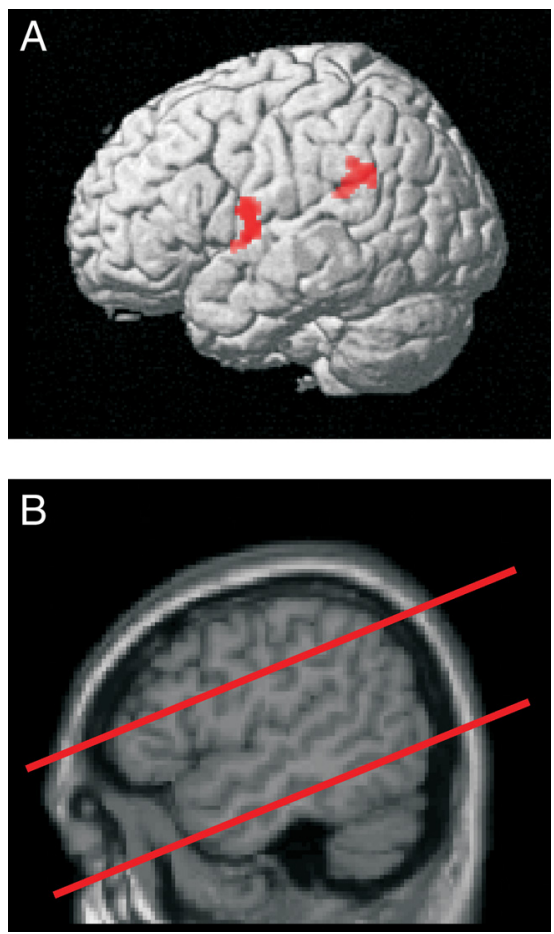

Figure 4. Group-level SPM. $\boldsymbol{A}$, This show the SPM showing where the xmRS effect was significant at the second level. The SPM has been thresholded liberally ( $p<0.05$ uncorrected at peak level and $p<0.1$ uncorrected at cluster level). This liberal threshold was chosen to show the spatial specificity of the effect. Peak level inference revealed significant peaks in two clusters. One was significant at $p<0.05$ corrected for small volume correction for left IFG; $t=6.35$ at $-50,-2,12$. The second was significant at $p<0.001$ uncorrected; $t=5.11$ at $-32,-48$, 30. This cluster could reflect activity in the anterior intraparietal sulcus (alPS) and has a similar location to BOLD activations reported in previous action observation studies (see Dinstein et al., 2007 for comparison). However, the scanning parameters of the current study did not allow for a total coverage of the aIPS, and therefore further studies would be required to confirm whether this did in fact correspond to activity alPS. For display the clusters are projected onto a rendered image of a template brain. $\boldsymbol{B}$, The position of the top and bottom slice showing the extent of the brain included in the 16 slices for a single subject.

during execution being more lateral than that when observing actions and the peak neural activity of mirror neurons (xmRS) being more ventral than that when observing actions.

\section{Discussion}

Although mirror neurons were discovered over a decade ago in the macaque monkey (di Pellegrino et al., 1992) there is still a lively debate as to whether any of the human neuroimaging studies constitute conclusive evidence for mirror neurons in the hu- 
Table 2. xmRS: same < different

\begin{tabular}{|c|c|c|c|c|c|c|c|c|c|c|}
\hline \multirow[b]{3}{*}{ Subject } & \multicolumn{5}{|c|}{ Left IFG } & \multicolumn{5}{|c|}{ Right IFG } \\
\hline & \multicolumn{3}{|c|}{ Peak MNI coordinates } & \multirow[b]{2}{*}{$p$ value } & \multirow[b]{2}{*}{$t$ value } & \multicolumn{3}{|c|}{ Peak MNI coordinates } & \multirow[b]{2}{*}{$p$ value } & \multirow[b]{2}{*}{$t$ value } \\
\hline & $x$ & $y$ & $Z$ & & & $x$ & $y$ & $Z$ & & \\
\hline 1 & -36 & 12 & 28 & 0.084 & 1.36 & 62 & 10 & 30 & 0.019 & 2.06 \\
\hline 2 & -50 & 8 & 18 & $<0.001$ & 5.5 & 58 & 12 & 22 & $<0.001$ & 3.21 \\
\hline 3 & -40 & 8 & 12 & 0.069 & 1.47 & 50 & -4 & 10 & 0.026 & 1.95 \\
\hline 4 & -56 & -4 & 16 & 0.007 & 2.48 & 46 & 8 & 18 & $<0.001$ & 3.49 \\
\hline 5 & -46 & 12 & 20 & 0.009 & 2.38 & 62 & 10 & 18 & $<0.001$ & 3.12 \\
\hline 6 & -58 & 8 & 24 & $<0.001$ & 3.48 & 62 & 2 & 24 & $<0.001$ & 3.75 \\
\hline 7 & -62 & -4 & 26 & 0.003 & 2.77 & 64 & -2 & 22 & $<0.001$ & 3.37 \\
\hline 8 & -62 & -2 & 20 & $<0.001$ & 4.01 & 64 & -4 & 36 & $<0.001$ & 3.19 \\
\hline 9 & -58 & 6 & 26 & $<0.001$ & 3.49 & 44 & 12 & 28 & $<0.003$ & 2.72 \\
\hline 10 & -60 & 12 & 22 & 0.002 & 2.83 & 58 & 12 & 36 & $<0.007$ & 2.44 \\
\hline
\end{tabular}

A

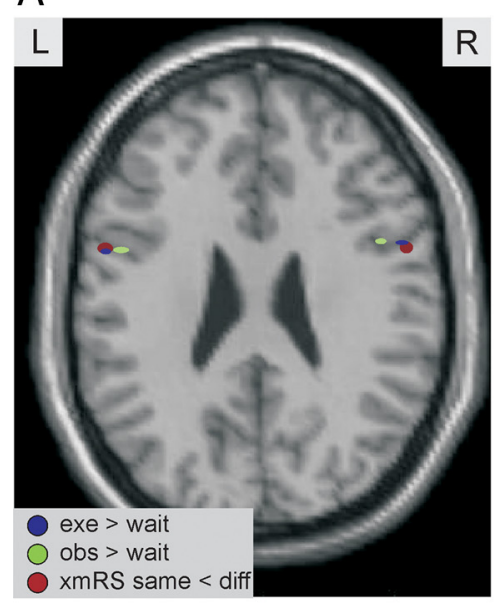

B
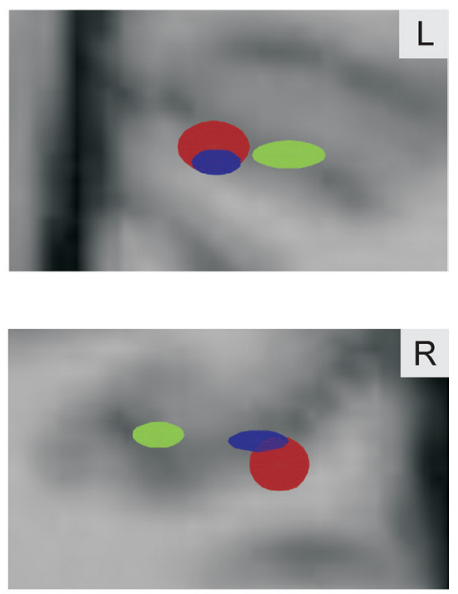

C

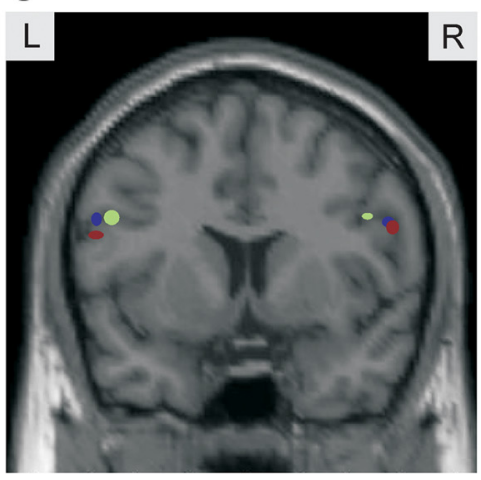

D
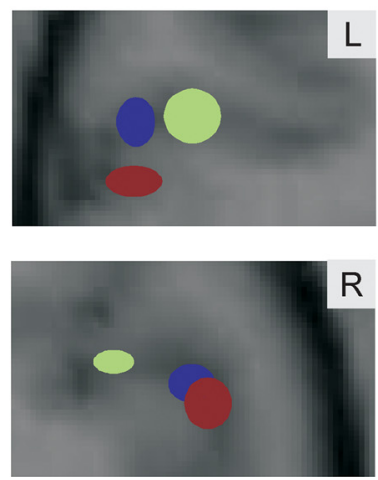

Figure 5. Spatial organization of IFG. $\boldsymbol{A}-\boldsymbol{D}$, The locations of the mean of the coordinates of the peak $t$ value for the three contrasts of interest: exe $>$ wait (blue), obs $>$ wait (green), and xmRS same $<$ different (red). In $\boldsymbol{A}$ and $\boldsymbol{B}$, the locations are depicted upon an axial slice and in $\boldsymbol{C}$ and $\boldsymbol{D}$ on a coronal slice. $\boldsymbol{B}, \boldsymbol{D}, \mathrm{A}$ magnified view of the data shown in $\boldsymbol{A}$ and $\boldsymbol{C}$, respectively. In each plot, the ellipsoid is centered at the mean coordinates across subjects, and the size of the ellipsoid is the SE in the $x-z$ coordinates.

man homolog of area F5, the IFG (Dinstein et al., 2007; Dinstein, 2008; Grafton, 2009; Lingnau et al., 2009). Here we adopted the fMRI technique of fMRI adaptation or repetition suppression to provide evidence of mirror neurons in the human IFG. Areas of the cortex that contain mirror neurons should show adaptation both when an action is first executed and subsequently observed and when an action is first observed and subsequently executed. Here we able to demonstrate these effects in human IFG, at the single-subject level, at the group level using an ROI analysis and also at the group level using a typical whole-brain analyses. Importantly we were able to demonstrate significant xmRS effects both when an observed action was followed by an executed action and vice versa. These results are consistent with the existence of mirror neurons in human IFG. This is evidence in favor of the existence of mirror neurons in what is considered the human homolog of area F5, IFG. We can assert this because the context established by the first withintrial stimulus (same or different) affects the respond to the second stimulus in a different modality (observation or execution). This influence can only be mediated by neuronal populations that respond to both observation and execution.

\section{Relationship to previous studies on xmRS}

Here we have demonstrated significant xmRS effects in the human IFG where three previous fMRI studies have failed (Dinstein et al., 2007; Chong et al., 2008; Lingnau et al., 2009;). There are a number of differences between the studies that might explain why the current study has yielded significant effects.

First, the task adopted here was designed to maximize the sensitivity to mirror-neuron discharge based on what is known of the properties of mirror neurons in area $\mathrm{F} 5$ of the macaque monkey. Here, the subjects executed and observed actions that involved grasping an object as opposed to the pantomime actions that were used in previous studies (Dinstein et al., 2007; Chong et al., 2008; Lingnau et al., 2009). Neurons in F5 of the macaque monkey have been shown to discharge predominantly during observation of actions with a goal (Umiltà et al., 2001; Rizzolatti and Craighero, 2004). Indeed, mirror neurons in the macaque have not been shown to discharge to mimed or pantomimed actions (Umiltà et al., 2001). Therefore, a priori there is no reason to expect mirror neurons to behave differently in humans. Therefore in the current study subjects observed and executed actions to actually physically 
grasp objects. However, not only did subjects perform and observe actual grasping actions but the objects grasped were carefully selected based on what is known of the firing properties of neurons in area F5 in the macaque monkey. The logic of the repetition suppression paradigm assumes that when the same stimulus is presented twice the same neuronal population is activated and when different stimuli are presented sequentially that different neuronal populations are activated. In other words, the success of the repetition suppression paradigm is contingent upon the different stimuli activating neuronal populations that are predominantly nonoverlapping. The two objects grasped in the current study were chosen because they have been shown in previous studies to activate different neuronal populations in area F5 of the macaque monkey (Gentilucci et al., 1988; Rizzolatti et al., 1988; Jeannerod et al., 1995). Given that the success of a repetition suppression paradigm is contingent upon the different stimuli activating neuronal populations that are predominantly nonoverlapping, it is of perhaps of no surprise that the effects at the individual subject level are focal, i.e., have high spatial specificity, even though the peak of the distribution within these clusters was often highly significant. This is similar to the fMRI results of Nelissen et al. (2005) that also demonstrated high spatial specificity of the BOLD response in area F5 of the macaque monkey.

Although the difference in the transitive nature of the action both executed and observed between the study described here and the three previous studies that failed to show xmRS could explain the difference in the results of the studies (Dinstein et al., 2007; Chong et al., 2008; Lingnau et al., 2009), the requirement for actual object grasping actions presents a potential interpretational issue (see Lingnau et al., 2009) — separating adaptation effects due to mirror neurons from those due to canonical neurons that respond to the object alone. Here we designed the manipulandum so that both objects were present in all observation trials. In this way subjects had no way of knowing which object was to be grasped, and the only difference between the repetitions was in the observed action and not in the objects presented. In this way we can assert that any xmRS effects observed here were not simply due to the discharge of object related neurons in IFG.

The second difference between the study described here and the three previous studies that failed to show xmRS in the IFG (Dinstein et al., 2007; Chong et al., 2008; Lingnau et al., 2009) was that in the current study, the scanning parameters were optimized to increase signal-to-noise ratio in the human IFG. Specifically, here we did not scan the whole brain but only scanned 16 slices that covered the IFG. This has the advantage that we collected approximately three times as much data from the IFG than we would have done had we collected whole-brain scans. However, this approach has the disadvantage that data were not collected from other regions of the cortex where previous studies have shown evidence for mirror neurons, such as the inferior parietal lobule (Fogassi et al., 2005; Hamilton and Grafton, 2006, 2008).

The final difference between the study described here and the three previous studies is that in the current study one of the three analyses that demonstrated a significant xmRS effect in the IFG was a group-level ROI analysis where the ROI was defined functionally and not anatomically. This is, we believe, an important factor. The human homolog of area F5 is not known. There is no probabilistic map of the human homolog of area F5 that one could define a priori. The human homolog of area F5 is known to contain areas of both BA44 and BA6 (Rizzolatti et al., 2001; Morin and Grèzes, 2008) and possibly even BA45. In other words, it is currently impossible to accurately define an ROI that corre- sponds to the areas of F5 in which mirror neurons have been found a priori. In light of this, we defined our ROI, a local weighted average centered on a single voxel, from the results of an orthogonal functional dataset (Note that defining an ROI based on functional data can be biased (Kriegeskorte et al., 2009). Here, the ROI defining contrast was orthogonal from the xmRS contrast and was even based on different columns of the same design matrix). The use of a subject-specific functionally defined ROI allowed for spatial variance in the xmRS response location within the IFG. All other analyses were restricted to a broad search region that included BA45, BA44, and BA6.

In addition to the uncertainty over the human homolog of area F5, area F5 has been shown to be subdivided into three cytoarchitecturally different regions-F5a, F5p, and F5c (Nelissen et al., 2005; Belmalih et al., 2009). Neurons in each of these subdivisions are activated under observation and execution of actions, but mirror neurons have been demonstrated only in area F5c (Rizzolatti and Craighero, 2004; Nelissen et al., 2005). It is therefore likely that the human homolog of area F5 is organized with similar subdivisions. Indeed here we show some evidence that this might be the case. We showed that the peaks of activity with IFG are in systematically different locations depending on whether the contrast was observing, executing, or xmRS (Fig. 5). These effects are small but significant. The different locations of the peak sensitivity to the different contrasts in area IFG reported here may correspond to such homologous subdivisions of human IFG. To date, human neuroimaging of mirror neuron activity has focused upon reporting activations with IFG at the group level, where these effects would not necessarily be observed, and as far as we are aware, no study has demonstrated subdivisions of IFG.

\section{Summary}

This study was designed to provide evidence that mirror neurons exist within the human homolog of monkey area F5, IFG. Previous studies demonstrating that fMRI activations have overlapping spatial representations within IFG are not sufficient evidence for human mirror neurons. Here we have shown that there is significant repetition suppression within IFG both when actions are first observed and then executed and vice versa. This is consistent with the behavior of mirror neurons in the monkey area F5. Furthermore we provide evidence that human IFG may contain subdivisions in a homologous manner to area F5 of the macaque monkey.

\section{References}

Belmalih A, Borra E, Contini M, Gerbella M, Rozzi S, Luppino G (2009) Cortical connections of the visuomotor parietooccipital area V6Ad of the macaque monkey. J Comp Neurol 512:183-217.

Buccino G, Binkofski F, Fink GR, Fadiga L, Fogassi L, Gallese V, Seitz RJ, Zilles K, Rizzolatti G, Freund HJ (2001) Action observation activates premotor and parietal areas in somatotopic manner: an fMRI study. Eur J Neurosci 13:400-404.

Chong TT, Cunnington R, Williams MA, Kanwisher N, Mattingley JB (2008) fMRI adaptation reveals mirror neurons in human inferior parietal cortex. Curr Biol 18:1576-1580.

Decety J, Grèzes J, Costes N, Perani D, Jeannerod M, Procyk E, Grassi F, Fazio F (1997) Brain activity during observation of actions. Influence of action content and subject's strategy. Brain 120:1763-1777.

Deichmann R, Schwarzbauer C, Turner R (2004) Optimisation of the 3D MDEFT sequence for anatomical brain imaging: technical implications at 1.5 and 3 T. Neuroimage 21:757-767.

Dinstein I (2008) Human cortex: reflections of mirror neurons. Curr Biol 18:R956-R959.

Dinstein I, Hasson U, Rubin N, Heeger DJ (2007) Brain areas selective for both observed and executed movements. J Neurophysiol 98:1415-1427. 
Dinstein I, Thomas C, Behrmann M, Heeger DJ (2008) A mirror up to nature. Curr Biol 18:R13-R18.

di Pellegrino G, Fadiga L, Fogassi L, Gallese V, Rizzolatti G (1992) Understanding motor events: a neurophysiological study. Exp Brain Res 91:176-180.

Fogassi L, Ferrari PF, Gesierich B, Rozzi S, Chersi F, Rizzolatti G (2005) Parietal lobe: from action organization to intention understanding. Science 308: 662-667.

Gallese V, Fadiga L, Fogassi L, Rizzolatti G (1996) Action recognition in the premotor cortex. Brain 119:593-609.

Gallese V, Fogassi L, Fadiga L, Rizzolatti G (2002) Action representation and the inferior parietal lobule. In: Attention and performance XIX. Common mechanisms in perception and action (Prinz W, Hommel B, eds), pp 334-355. Oxford: Oxford UP.

Gazzola V, Keysers C (2009) The observation and execution of actions share motor and somatosensory voxels in all tested subjects: single-subject analyses of unsmoothed fMRI data. Cereb Cortex 19:1239-1255.

Gentilucci M, Fogassi L, Luppino G, Matelli M, Camarda R, Rizzolatti G (1988) Functional organization of inferior area 6 in the macaque monkey. I. Somatotopy and the control of proximal movements. Exp Brain Res 71:475-490.

Grafton ST (2009) Embodied cognition and the simulation of action to understand others. Ann N Y Acad Sci 1156:97-117.

Grèzes J, Decety J (2001) Functional anatomy of execution, mental simulation, observation, and verb generation of actions: a meta-analysis. Hum Brain Mapp 12:1-19.

Grill-Spector K, Henson R, Martin A (2006) Repetition and the brain: neural models of stimulus-specific effects. Trends Cogn Sci 10:14-23.

Hamilton AF, Grafton ST (2006) Goal representation in human anterior intraparietal sulcus. J Neurosci 26:1133-1137.
Hamilton AF, Grafton ST (2008) Action outcomes are represented in human inferior frontoparietal cortex. Cereb Cortex 18:1160-1168.

Jeannerod M, Arbib MA, Rizzolatti G, Sakata H (1995) Grasping objects: the cortical mechanisms of visuomotor transformation. Trends Neurosci 18:314-320.

Kriegeskorte N, Simmons WK, Bellgowan PS, Baker CI (2009) Circular analysis in systems neuroscience: the dangers of double dipping. Nat Neurosci 12:535-540

Lingnau A, Gesierich B, Caramazza A (2009) Asymmetric fMRI adaptation reveals no evidence for mirror neurons in humans. Proc Natl Acad Sci U S A 106:9925-9930.

Morin O, Grèzes J (2008) What is "mirror" in the premotor cortex? A review. Neurophysiol Clin 38:189-195.

Nelissen K, Luppino G, Vanduffel W, Rizzolatti G, Orban GA (2005) Observing others: multiple action representation in the frontal lobe. Science 310:332-336.

Rizzolatti G, Craighero L (2004) The mirror-neuron system. Annu Rev Neurosci 27:169-192.

Rizzolatti G, Camarda R, Fogassi L, Gentilucci M, Luppino G, Matelli M (1988) Functional organization of inferior area 6 in the macaque monkey. II. Area F5 and the control of distal movements. Exp Brain Res 71:491-507.

Rizzolatti G, Fadiga L, Matelli M, Bettinardi V, Paulesu E, Perani D, Fazio F (1996) Localization of grasp representations in humans by PET: 1. Observation versus execution. Exp Brain Res 111:246-252.

Rizzolatti G, Fogassi L, Gallese V (2001) Neurophysiological mechanisms underlying the understanding and imitation of action. Nat Rev Neurosci 2:661-670.

Umiltà MA, Kohler E, Gallese V, Fogassi L, Fadiga L, Keysers C, Rizzolatti G (2001) I know what you are doing. A neurophysiological study. Neuron 31:155-165. 